\title{
Measuring the daily stepping activity of people with transtibial amputation using the activePAL ${ }^{\mathrm{TM}}$ activity monitor.
}

\author{
Arjan Buis, Ph.D Kevin D Murray, PhD Sandra Sexton, Bsc Tim Dumbleton, $\mathrm{PhD}$ \\ Geoff McKay, PhD Brendan F McHugh, PhD. \\ University of Strathclyde, Glasgow, UK
}

\begin{abstract}
This study compared the general activity over one week and detailed activity during a 24-hour period, of 48 established unilateral transtibial prostheses users. Activity was measured by instrumenting their prescribed prosthesis, which they have been using for a minimum of 6 month, with the ActivPAL ${ }^{\mathrm{TM}}$ activity monitor.

Half $(n=24)$ were fitted with a prostheses with total surface bearing (TSB) pressurecast sockets (Hands-off) and the other half $(n=24)$ had been wearing prostheses with hand-cast (Hands-on) patella tendon bearing (PTB) sockets. As a prerequisite, the long-term reliability of the ActivPAL ${ }^{\mathrm{TM}}$ activity monitor was assessed and it was found to exhibit a high level of consistency between devices (Interclass Correlation Coefficient (ICC) of 0.997 over a 24 -hour period). The monitors were utilized to examine the activity levels of two groups of transtibial prostheses users wearing their own prosthesis. Results indicated that both subject groups were active throughout the day, walking on average over 8,000 steps. No statistically significant difference in daily stepping activity was seen between the two groups $(\mathrm{p}=0.173)$. Despite differences in prosthetic socket design the daily activity profiles of both subject groups were similar.
\end{abstract}

\section{INTRODUCTION}

The purpose of this study was to investigate the general activity during 1 week and detailed activity during a 24-hour period, of 48 established unilateral transtibial prostheses users instrumented with an active PAL ${ }^{\mathrm{TM}}$ activity monitor. Physical activity is inversely associated with disease and is therefore important for the health and well-being of people of all ages and abilities. ${ }^{1,2}$ This is equally true of people with leg amputation associated with vascular disease and/or diabetes and have reduced life expectancy. ${ }^{3,4}$ In addition, adults with transtibial amputation (TTAs) are believed to be generally less active than persons without a lower limb amputation 5-7, especially vascular TTAs (where amputation was the result of vascular disease associated with conditions such as diabetes or atherosclerosis $)^{7,8}$. Therefore, ongoing mobility can be an important indicator of the quality of prosthetic treatment. ${ }^{6,8}$

Prosthetists are able to observe the individual walking within the clinical environment. Outside that environment, prosthetic users can report on their activity by completing a questionnaire or responding to interview questions. Although these methods are quick and inexpensive, they only provide subjective data and can be difficult to analyze i.e. respondents might not be completely truthful with the supplied information for various reasons such as, private, a specific component prescription and or range supplied, to name a few. Therefore the accuracy of such subjective measures may sometimes be limited. ${ }^{9,10}$ As a result there is increasing interest in more objective methods of assessing prosthetic use beyond the clinic 
room. ${ }^{11}$ Portable instrumentation for monitoring the daily activity of a person with transtibial limb loss is one potential solution for objectively recording the level of prosthetic use. $^{6-8,11}$

\section{METHODS}

The investigation was implemented following ethical approval granted by the Local Regional Health Authority and University Ethics Committees (Ref EC/03/S/66). We report on an investigation regarding prosthetic users' activity levels. This was part of a larger study including interface pressure measurement within the prosthetic socket and the use of a questionnaire to gauge the subject's satisfaction with their prosthesis. The findings related to the other outcome measures, interface socket pressure and user satisfaction, have been be reported separately. ${ }^{12}$ A total of 133 prosthetic users from the West of Scotland Mobility and Rehabilitation Centre (WESTMARC), based at the Southern General Hospital situated in Glasgow, were invited to participate in the overall study. The participants recruited had established unilateral TT amputation of at least one year and had been wearing their current prosthesis on a daily basis for at least 6 months. From 79 people who responded positively two balanced groups of 24 subjects, were selected for participation in the study. One group has been using hand cast PTB sockets with supracondylar suspension and pressure cast (using Ice Cast Compact TM) TSB sockets with pin-lock silicon liners respectively.

The authors are aware that there are other aspects of prosthesis design that can influence mobility and activity levels, i.e. Suspension, alignment, ankle-foot mechanism, footwear combination, heel height, rocker profile etc. However, this activity study reports if there are differences in steps taken over a defined period with prosthesis supplied with two distinct different socket designs.

In this study, the activePAL ${ }^{\mathrm{TM}}$ monitor (PAL Technologies Ltd, Glasgow, Scotland, UK) was used to record the daily activity of all 48 participants. The activePAL ${ }^{\mathrm{TM}}$ activity monitor is a small, lightweight and discrete monitor. Figure 1.

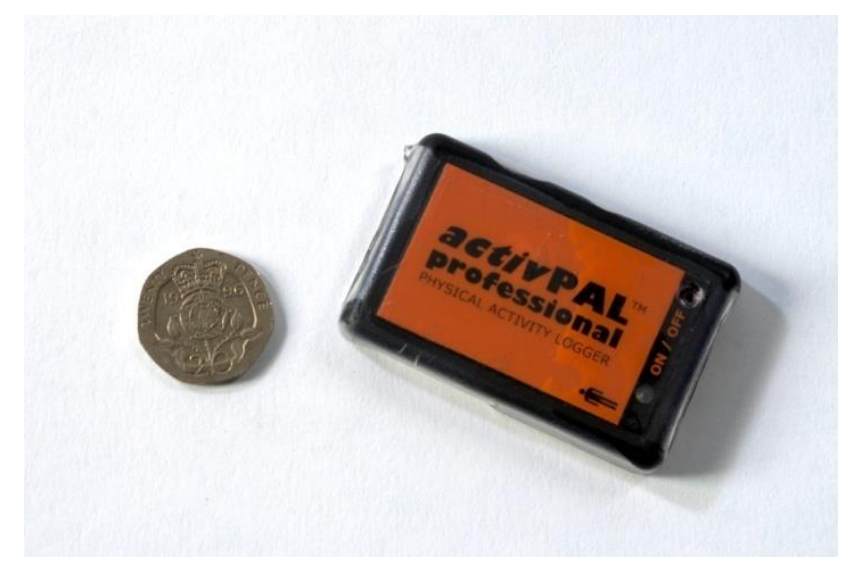

Figure 1: The activePALTM physical activity monitor aspects.

The acceleration of the leg is recorded at a sampling rate of the monitors is $10 \mathrm{~Hz}$.

The monitors are extremely lightweight, weighing about $20 \mathrm{~g}$ including the battery 
making them ideal for placement on the subject's prosthesis, where weight considerations are important. Figure 2.

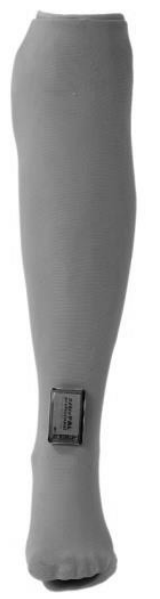

Figure 2: In situ location of the activePAL TM monitor.

The small size of the monitors means that they do not interfere with daily activities and are not obtrusive through clothing. Each monitor is approximately $53 \mathrm{~mm}$ long, $35 \mathrm{~mm}$ wide and $7 \mathrm{~mm}$ thick, Operation of the monitor is conducted via a $3 \mathrm{~V}$ lithium Ion battery providing sufficient power for the recording period. The memory capacity of the monitor is capable of recording daily activity for periods of over a week. Subjects wore the monitor for one week during the investigation; therefore the memory was sufficient for the requirements of this study.

The active PAL ${ }^{\mathrm{TM}}$ monitors provide information regarding the number of steps taken each day, the cadence and the time of day the activity was performed. The active PAL ${ }^{\mathrm{TM}}$ software displays the recording output in various forms. Outputs can be displayed either as a summary of daily activities or by hourly activity. The summary of daily activity shows activity on an hour by hour basis, indicating the proportion of each of the three forms of usage, i.e. walking, standing or sitting. However, due to the position of the monitor in this study, only the stepping activity is used, as the orientation of the monitor could not detect whether the subject was sitting or standing.

Grant et al ${ }^{13}$ reported on the validation of the activePAL ${ }^{\text {TM }}$ monitors. Calculations of the inter-device reliability using ICC $(2,1)$ were made from the data recorded by three active $\mathrm{PAL} \mathrm{L}^{\mathrm{TM}}$ activity monitors during a series of tests. Those test included 10 healthy volunteers a controlled section and an activities of daily living (ADL) were participants performed six everyday activities in a random order. Each testing section lasted 15-20 min. It was concluded that the inter-device reliability was good to excellent $(\operatorname{ICC}(2,1)=0.79$ to 0.99$)$. An ICC value of $\geqslant 0.75$ was considered good and $\geqslant 0.9$ was deemed excellent.

A similar study described by Ryan et $\mathrm{al}^{14}$ investigated the validity and reliability of the active PAL ${ }^{\mathrm{TM}}$ monitor system, with the objective to investigate the validity and reliability of the active PAL ${ }^{\mathrm{TM}}$ activity monitor in measuring step number and cadence. It was reported that step number and cadence in 20 healthy adults (age 
34.5+/-6.9 years; BMI 26.8+/-4.8 (mean+/-SD)) was evaluated against video observation. The participants were asked to walk at five different speeds $(0.90,1.12$, $1.33,1.56$, and $1.78 \mathrm{~m} / \mathrm{s}$ ) on a treadmill. In addition, outdoor tests were performed at three self-selected walking speeds (slow, normal, and fast). It was reported that for all speeds, inter device reliability was excellent $(\operatorname{ICC}(2,1)>$ or $=0.99)$ for both step number and cadence. It was found that the absolute percentage error for the active PAL ${ }^{\mathrm{TM}}$ was $<1.11 \%$ for step number and cadence regardless of walking speed. They concluded that the activity monitor is a valid and reliable measure of walking in healthy adults and that accuracy is not influenced by walking speed.

Eight activePAL ${ }^{\mathrm{TM}}$ monitors were utilized in our study. The objective was to use the active $\mathrm{PAL}^{\mathrm{TM}}$ monitor to record the daily activity of prosthetic limb users over a period of 7 days and then analyze in detail the activity over the 24-hour time period which most closely represented the average daily number of steps. Before proceeding with this task, a preliminary study was carried out to corroborate the findings of the aforementioned previous studies and to confirm that the monitors performed consistently over an extended period of continuous use.

\section{Preliminary Study of Long-term Reliability of activePAL ${ }^{\mathrm{TM}}$ Activity Monitor}

For the preliminary study a healthy adult subject was chosen to wear two monitors selected at random from the eight available for the main clinical trial. The two randomly selected monitors were placed in a specially designed neoprene cuff manufactured to fit around the subject's lower leg at a position just above the ankle. Each cuff had space to securely accommodate one pair of monitors. The monitors were stacked on top of each other. Thus both monitors were positioned at the same height from the ground, and in the same orientation. The subject wore each pair of monitors for 24 hours whilst performing normal daily activities. A total of 20 trials were completed.

\section{Daily Activity of persons with transtibial limb loss}

In total 48 subjects participated in the study, Table 1. All subjects wore their own transtibial prosthesis. Two sub groups were established for comparison purposes. One group wore a "hands on" prosthetic socket concept. These sockets had been hand cast by the prosthetist. The second group wore "hands off" sockets. These had been cast using a uniform pressure technique. Each of the prosthesis had been worn by the user for at least six months prior to recording the daily activity.

\begin{tabular}{|l|l|l|l|l|l|}
\hline Socket & $\begin{array}{l}\text { No. of } \\
\text { Subjects }\end{array}$ & $\begin{array}{l}\text { Gender } \\
\text { (Male/Female) }\end{array}$ & $\begin{array}{l}\text { Age } \\
\text { (SD) }\end{array}$ & $\begin{array}{l}\text { BMI } \\
\text { (SD) }\end{array}$ & $\begin{array}{l}\text { Reason for } \\
\text { Amputation } \\
\text { (PVD/Other) }\end{array}$ \\
\hline Hands Off & 24 & $20 / 4$ & $50.04(11.89)$ & $27.63(4.99)$ & $4 / 20$ \\
\hline Hands On & 24 & $20 / 4$ & $60.54(14.85)$ & $28.52(5.44)$ & $8 / 16$ \\
\hline
\end{tabular}

Table 1: Subject Demographic 
During this investigation the monitor was positioned at the level of the ankle, on the anterior aspect of the prosthesis, securely attached using strong tape, Figure 2.

The small size and light weight of the monitors makes this a suitable position for placement on the prosthesis. Once placed in position and switched on by the researcher, the monitor could be left in this location, with no need for the subject to touch it for the duration of the recording. The monitor was attached below the sock level, therefore was normally concealed.

Locating the monitor at the ankle presented benefits for the participant and for the reliability of the data. The subject would not continually be reminded of the monitor, and would not have to remove it each time they bathed. The reliability of the data could be maintained as the monitor position could be checked by the researcher before the trial began, and would remain in the same location for the duration of the recording.

\section{RESULTS}

\section{Long-term reliability of activePAL ${ }^{\mathrm{TM}}$ Activity Monitor}

The inter-device reliability of the monitors was determined using intra-class correlation coefficient (ICC 2,1) analysis. The ICC value was calculated to be 0.997 , indicating excellent reliability over the 24-hour time period.

This confirmed the longer term reliability of the ActivPAL ${ }^{\mathrm{TM}}$ monitor. Analysis of the data obtained showed a high degree of consistency and confirmed the shorter term findings of Grant et al. ${ }^{13}$ This reliability would be important when comparing recordings from subjects over a 24 -hour period.

\section{Transtibial prosthetic user activity Number of Steps Taken}

Each subject wore the monitor for a maximum of six days (complete 24-hour periods) of continuous activity. The average (mean) daily number of steps taken and percentage time spent walking by each subject were calculated and used for the purposes of analysis, Table 2 .

\begin{tabular}{|l|l|l|l|l|}
\hline $\begin{array}{l}\text { Socket } \\
\text { Concept }\end{array}$ & $\begin{array}{l}\text { Number of Steps } \\
\text { Taken per Day } \\
\text { (SD) }\end{array}$ & Range & $\begin{array}{l}\% \text { Time Spent in } \\
\text { Walking Activity } \\
\text { (SD) }\end{array}$ & Range \\
\hline $\begin{array}{l}\text { Hands } \\
\text { Off }\end{array}$ & $9130.024(4420)$ & $1570-16221$ & $7.525(3.719)$ & $1.5-14.3$ \\
\hline Hands On & $7383.21(4383)$ & $1601-16815$ & $6.154(3.272)$ & $1.7-12.7$ \\
\hline
\end{tabular}

Table 2: Daily Activity 
Table 2 shows that subjects wearing the hands off prosthesis walked on average 9130 (SD 4420) steps per day and the group wearing the hands on sockets walked on average 7383 (SD 4383) steps. There was a wide variation in the number of steps between the subjects which can be seen when examining the standard deviation of the two groups. A two sample independent t-test (alpha level 0.05) was used to check the difference in daily steps taken between the two groups. Results of this test show that there is no statistically significant difference in the steps taken between the two casting groups $(\mathrm{p}=0.173)$.

In the analysis of the sample groups it was seen that the average age of the two groups differed by 10 years. This difference was consistent with the clinical populations from which the participants were recruited. A Pearson correlation test was performed on both groups between age and number of steps was taken for each group to determine if the age of the subject has any relationship to the number of steps taken., Results indicated no significant relationship between age and steps taken for either group (Hands Off group $p=0.409 \mathrm{r}=-0.177$, Hands On group $\mathrm{p}=0.879$ $\mathrm{r}=-0.033)$.

\section{Time Spent in Walking Activity}

In addition to the average number of steps taken each day, the activity monitors provide the total time spent stepping. This is displayed in terms of hours, and percentage of total time. For comparison, the percentage of time spent walking is used. Table 2 shows the results. Independent sample t-tests show that no significant differences exist between casting groups $(\mathrm{p}=0.182)$.

The activity monitors provide information as to the cadence and time of activity. For the purposes of analysis, one particular day from all those recorded by each subject was selected for analysis of cadence and time of activity. The criterion for selection was that the total number of steps taken on that day most closely matched the average for that subject over the six days monitored.

The average cadence for each sample group is displayed in the graph in Figure 3. The time of day of Activity of each group is shown in Figure 4.

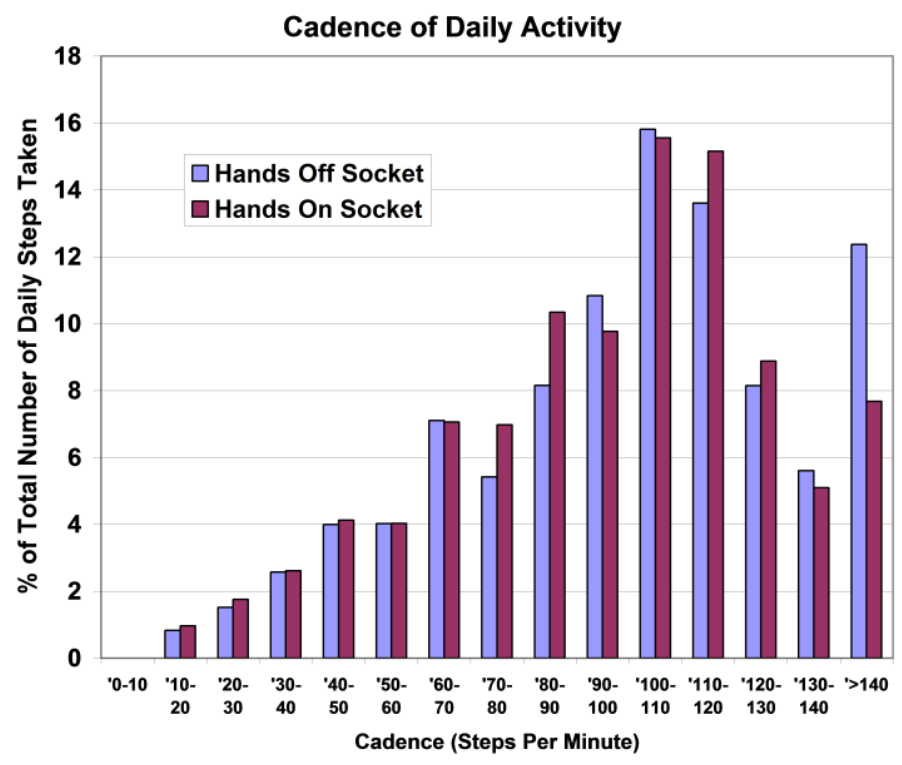




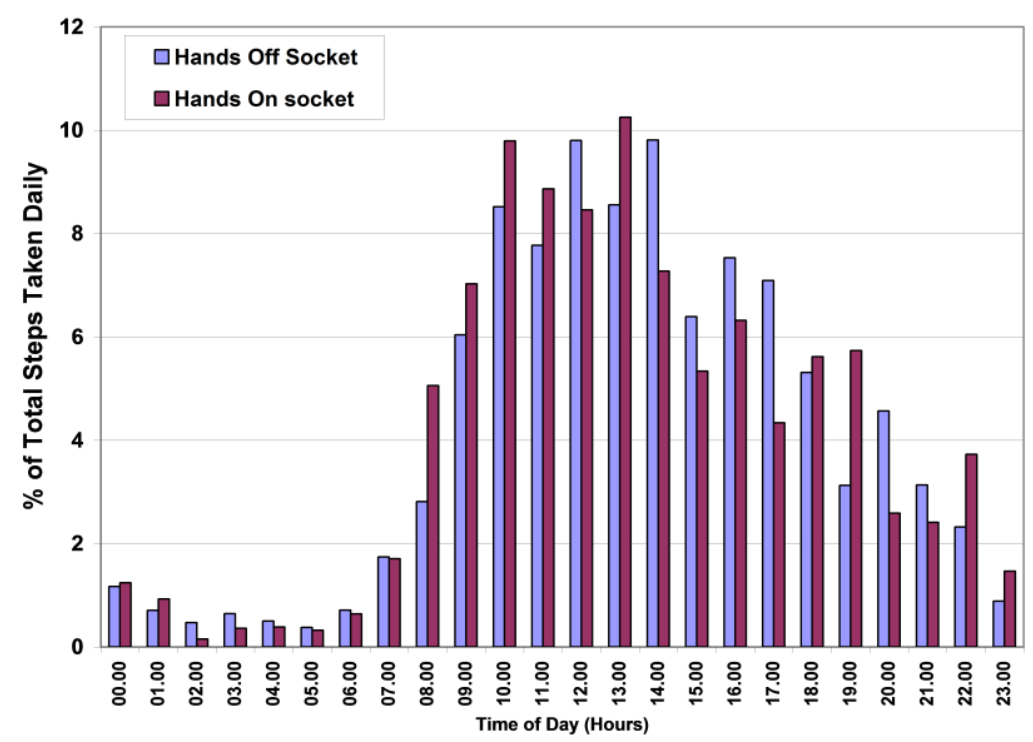

Figure 3: The average cadence for each sample group. Figure 4: Activity of each group during a 24 hour period.

\section{DISCUSSION}

Results from activity monitoring indicate that, on average, the number of steps taken on a daily basis by subjects wearing the hands off sockets is 9130 . The group wearing the hands on sockets walked on average 7383 steps. These results would suggest that subjects in the group wearing the hands off sockets are the more active. However, because of the large variation within the two groups there is no statistically significant difference between them $(\mathrm{p}=0.173)$. The range of daily activity, around 1,500 steps to 17,000 steps, was similar for both groups. These results imply that the design of prosthetic socket does not have a major effect the daily activity of the user. In addition, the results highlight the fact many of the subjects remain highly active after amputation.

According to a review of 32 published studies of physical activity measurement, healthy older adults can be expected to take 6,000-8,500 steps/day while individuals with disabilities and chronic illnesses are restricted to 3,500-5,500 steps/day ${ }^{15}$. Thus both groups in our study performed, on average, at the level of normal adults of similar age. This, on itself, is one indicator of the success of prosthetic treatment.

The percentage of the day spent walking by each subject was $6.67 \%$. No significant difference exists between the two socket concepts for the length of time spent walking. The profile for walking was very similar for both groups. Walking activity was spread throughout the day, with most activity around late morning and early afternoon. The output from the activity monitors indicated that although subjects only spent around 100 minutes per day in walking activity, the prostheses were in use for more than 8 hours per day albeit sometimes sporadically. The graph in Figure 3 and Figure 4 indicates that both groups have a similar cadence. The most 
common cadence of between $100 \mathrm{steps} / \mathrm{min}$ and $110 \mathrm{steps} / \mathrm{min}$ is achieved in nearly $16 \%$ of all steps taken.

It should be noted that the authors limited their assessment of walking to only a few variables but that additional variables are known to influence walking (i.e., body mass, occupation, living environment, prosthesis components and design features, etc.). In addition a prerequisite for inclusion to this study, subjects had been using their prosthesis for at least six months for daily activities, with no health issues in relation to their residual limb. The subject groups also included a high proportion of people with traumatic amputations. This was beyond the control of the researchers and was due to the category of prosthetic users willing to participate in the study. This may account for the relatively high daily activity levels recorded in this study. Daily activity prior to amputation had not been recorded; however the study does provide encouraging evidence that a high level of daily activity can be maintained after amputation.

\section{CONCLUSION}

In order to better evaluate how a prosthesis is functioning there is a need to examine users outside the confines of the clinic room. Activity monitors have been shown to be a relative simple and reliable tool for measuring outcomes of prosthetic intervention without interfering with activities of daily living.

The authors limited their assessment of walking to only a few variables but that additional variables are known to influence walking (i.e., body mass, BMI, occupation, living environment, prosthesis components and design features, etc.)

The two socket concepts used in this study would be expected to interact differently with the residual limb in terms of pressure distribution and stability. However, the results of the analysis indicate that the differences in design do not result in significant differences in activity level.

Furthermore, the mean activity level for each design is within the range of normality for the age group concerned. This would suggest that both methods of manufacturing a prosthetic socket can provide a satisfactory outcome in terms of activity level. It further emphasizes that persons with a transtibial amputation and wearing a well-fitting prosthesis can maintain activity levels equivalent to those of their non-amputated peer group.

Acknowledgements;

This study was funded by Action Medical Research, grant reference AP0985. The authors would like to thank Dr. Angus McFadyen, Glasgow Caledonian University for his help in analysing the data.

Conflict of interest;

None declared. 


\section{REFERENCES}

1. Kesaniemi YA, Danforth E, Jensen MD, et al. Dose-response issues concerning physical activity and health: An evidence-based symposium. Medicine and science in sports and exercise. 2001; 33: 531-8.

2. Blair S, Cheng Y and Holder JS. Is physical activity or physical fitness more important in defining health benefits? Medicine and science in sports and exercise. 2001; 33: 379-99.

3. Barth DG, Schumacher L and Thomas SS. Gait analysis and energy cost of below-knee amputees wearing six different prosthetic feet. Journal of Prosthetics and Orthotics. 1992; 4: 63-75.

4. Bo Ebskov L. Dysvascular amputations and long-term survival in a 20-year follow-up study. International Journal of Rehabilitation Research. 2006; 29: 325-8.

5. Morrato EH, Hill JO, Wyatt HR, et al. Physical activity in U.S. adults with diabetes and at risk for developing diabetes, 2003. Diabetes Care. 2007; 30: 203-9.

6. Bussmann JB, Schrauwen HJ and Stam HJ. Daily physical activity and heart rate response in people with a unilateral traumatic transtibial amputation. Archives of Physical Medicine and Rehabilitation. 2008; 89: 430-4.

7. Van den Berg-Emons RJ, Bussmann JB and Stam HJ. Accelerometry-based activity spectrum in persons with chronic physical conditions Archives of Physical Medicine and Rehabilitation. 2010; 91: 1856-61

8. Bussmann JB, Grootscholten EA and Stam HJ. Daily physical activity and heart rate response in people with a unilateral transtibial amputation for vascular disease. Archives of Physical Medicine and Rehabilitation. 2004; 85: 240-4.

9. Sager MA, Dunham NC, Schwanters A, et al. Measurement of activities of daily living in hospitalized elderly: a comparison of self-report and performance-based methods. J Am Geriatr Soc. 1992; 40: 457-62.

10. Smith DG, Domholdt E, Coleman KL, et al. Ambulatory activity in men with diabetes: Relationship between self-reported and real-world performance-based measures. J Rehabil Res Dev. 2004; 41: 571-9.

11. Thomas JR, Nelson JK and Silverman SJ. Research methods in physical activity. 6th ed. Champaign, IL: Human Kinetics, 2011.

12. Dumbleton T, Buis AW, McFadyen A, et al. Dynamic interface pressure distributions of two transtibial prosthetic socket concepts. $J$ Rehabil Res Dev. 2009; 46: 405-15.

13. Grant PM, Ryan CG, Tigbe WW and Granat MH. The validation of a novel activity monitor in the measurement of posture and motion during everyday activities. Br J Sports Med 2006; 40: 992-7.

14. Ryan CG, Grant PM, Tigbe WW and Granat MH. The validity and reliability of a novel activity monitor as a measure of walking. Br J Sports Med 2006; 40: 779-84.

15. Tudor-Locke C, Sisson SB, Collova T, Lee SM, et al. Pedometer-determined step count guidelines for classifying walking intensity in a young ostensibly healthy population. Canadian Journal of Applied Physiology. 2005; 30: 66676.

16. Waters, R.L., et al., Energy cost of walking of amputees: The influence of level of amputation. The Journal of Bone \& Joint Surgery, 1976. 58: p. 4246. 